\title{
The Integration of Functional Brain Activity from Adolescence to Adulthood
}

\author{
Prantik Kundu, ${ }^{1}{ }^{\circledR}$ Brenda E. Benson, ${ }^{3}$ Dana Rosen, ${ }^{4}$ Sophia Frangou, ${ }^{2}$ Ellen Leibenluft, ${ }^{3}$ Wen-Ming Luh, ${ }^{6}$ \\ -Deter A. Bandettini, ${ }^{5}$ Doniel S. Pine, ${ }^{3}$ and Monique Ernst ${ }^{4}$ \\ ${ }^{1}$ Section on Advanced Functional Neuroimaging, Brain Imaging Center, ${ }^{2}$ Department of Psychiatry, Icahn School of Medicine at Mount Sinai, New York, \\ New York 10029, ${ }^{3}$ Emotion and Development Branch, ${ }^{4}$ Section on Neurobiology of Fear and Anxiety, ${ }^{5}$ Section on Functional Imaging Methods, National \\ Institute of Mental Health, Bethesda, Maryland 20892, and ${ }^{6}$ Cornell MRI Facility, Cornell University, Ithaca, New York 14853
}

Age-related changes in human functional neuroanatomy are poorly understood. This is partly due to the limits of interpretation of standard fMRI. These limits relate to age-related variation in noise levels in data from different subjects, and the common use of standard adult brain parcellations for developmental studies. Here we used an emerging MRI approach called multiecho (ME)-fMRI to characterize functional brain changes with age. ME-fMRI acquires blood oxygenation level-dependent (BOLD) signals while also quantifying susceptibility-weighted transverse relaxation time $\left(\mathrm{T}_{2}^{*}\right)$ signal decay. This approach newly enables reliable detection of BOLD signal components at the subject level as opposed to solely at the group-average level. In turn, it supports more robust characterization of the variability in functional brain organization across individuals. We hypothesized that BOLD components in the resting state are not stable with age, and would decrease in number from adolescence to adulthood. This runs counter to the current assumptions in neurodevelopmental analyses of brain connectivity that the number of BOLD signal components is a random effect. From resting-state ME-fMRI of 51 healthy subjects of both sexes, between 8.3 and 46.2 years of age, we found a highly significant $(r=-0.55, p \ll 0.001)$ exponential decrease in the number of BOLD components with age. The number of BOLD components were halved from adolescence to the fifth decade of life, stabilizing in middle adulthood. The regions driving this change were dorsolateral prefrontal cortices, parietal cortex, and cerebellum. The functional network of these regions centered on the cerebellum. We conclude that an age-related decrease in BOLD component number concurs with the hypothesis of neurodevelopmental integration of functional brain activity. We show evidence that the cerebellum may play a key role in this process.

Key words: complexity; development; fMRI; multiecho; resting state

\section{Significance Statement}

Human brain development is ongoing from childhood to at least 30 years of age. Functional MRI (fMRI) is key for characterizing changes in brain function that accompany development. However, developmental fMRI studies have relied on reference maps of adult brain organization in the analysis of data from younger subjects. This approach may limit the characterization of functional activity patterns that are particular to children and adolescents. Here we used an emerging fMRI approach called multi-echo fMRI that is not susceptible to such biases when analyzing the variation in functional brain organization over development. We hypothesized an integration of the components of brain activity over development, and found that the number of components decreases exponentially, halving from 8 to 35 years of age. The brain regions most affected underlie executive function and coordination. In summary, we show major changes in the organization and integration of functional networks over development into adulthood, with both methodological and neurobiological implications for future lifespan and disease studies on brain connectivity.

\section{Introduction}

Characterizing brain development from adolescence to adulthood is critical for understanding neuropsychiatric disease and

Received June 27, 2017; revised Jan. 21, 2018; accepted Jan. 25, 2018.

Author contributions: P.K., B.E.B., S.F., E.L., W.-M.L.,P.A.B., D.S.P., and M.E. designed research; P.K., B.E.B., D.R., W.-M.L., D.S.P., and M.E. performed research; P.K. contributed unpublished reagents/analytic tools; P.K., B.E.B., E.L., W.-M.L., and M.E. analyzed data; P.K., B.E.B., S.F., E.L., W.-M.L., P.A.B., D.S.P., and M.E. wrote the paper.

Correspondence should be addressed to Prantik Kundu, Section on Advanced Functional Neuroimaging, Brain Imaging Center, Icahn School of Medicine at Mount Sinai, New York, NY 10029. E-mail: prantikk@gmail.com or prantik.kundu@mssm.edu. healthy brain function. However, the trajectories of changes in functional organization during brain development are not yet well characterized. Developmental studies of white matter structural change based on diffusion weighted MRI report nonlinear trajectories involving faster changes at earlier ages, followed by stabilization at later ages (Wallace et al., 2006). Microstructural changes in white matter are also known to be 
tract and region specific, suggesting that trajectories are both global and regionally specific (Paus et al., 2001). In this study, we use advanced functional MRI (fMRI) techniques to address age-related trajectories of change in functional brain organization at both the whole-brain and regional levels.

Recent findings indicate that neurodevelopmental changes in the organization of functional networks are detectable as agerelated increases in network coherence (Gu et al., 2015). It follows that networks of functional correlation may become more integrated from adolescence into adulthood, changing the broader organization of functional networks. However, age-related differences in anatomy complicate the comparison of functional activity data across development (Power et al., 2012). To more sensitively detect trajectories of age-related functional brain change, we used multiecho (ME) fMRI (Kundu et al., 2015) to image the resting state. ME-fMRI isolates the susceptibility-weighted transverse relaxation $\left(\mathrm{T}_{2}^{*}\right)$ component of blood oxygenation leveldependent (BOLD) fMRI signals without many of the arbitrary denoising models used for standard fMRI. Furthermore, using ME-fMRI, BOLD signal components can be characterized without standard brain parcellations derived from adult data, which are commonly used, but may bias results to represent normative adult anatomy (Power et al., 2011; Craddock et al., 2012).

In this study, we especially focused on characterizing the number of functional BOLD components in the resting state as a marker of brain development. The number of BOLD components in resting-state fMRI data may be considered to represent the dimensionality, degrees of freedom, or dimensionality of spontaneous brain activity (Friston et al., 1995). We considered that this parameter could be useful in representing the level of fragmentation versus the integration of functional networks across the brain. The variation of BOLD component number with age is evaluated in this article. Specifically, we had the following three aims: (1) to show that age affects the number of components in the fMRI signal, as detected experimentally using ME-independent component analysis (ICA); (2) to define datadriven brain regions that are particularly susceptible to the impact of age in terms of the number of BOLD components they express; and (3) to characterize the relationships among brain regions that show similar trajectories of change in regional BOLD component number. Altogether, we find that functional brain networks undergo a trajectory of functional integration with age, in a regionally specific way, which has implications for the development of normal cognition and behavior as well as neurodevelopmental disorders.

\section{Materials and Methods}

\section{Overview}

We assessed three levels of functional brain organization. First, we determined the number of BOLD signal components at the individual subject level. Second, for each subject, we computed a map of the number of components corepresented in each voxel. Third, we computed the relationship between component corepresentation and subject age, yielding regions of interest (ROIs) of age-related component change. We then used these ROIs as seed regions to estimate their functional connectivity and elucidate age-sensitive functional networks.

\section{Experimental design}

Participants. Fifty-one healthy subjects (mean age, 21.9 years; age range, 8.3-46.2 years; 20 females) completed the study. This study was approved by the National Institutes of Health Institutional Review Board.

Image acquisition. Data were acquired on a GE MR750 3 T Scanner using a 32-channel receive-only head coil (GE Healthcare). Each imaging session first involved acquiring a whole-brain anatomical MPRAGE scan with $1 \mathrm{~mm}$ isotropic resolution. The resting-state fMRI scan was $10 \mathrm{~min}$ long and involved acquisition of multiecho time-course EPI using the following parameters: $240 \mathrm{~cm}$ field of view; $64 \times 64$ resolution yielding 3.75 isotropic voxels; in-plane SENSE (sensitivity encoding) acceleration factor, 2; flip angle, $77^{\circ}$; repetition time (TR), $2.0 \mathrm{~s}$; and echo times (TEs), $12.8,28$, and $43 \mathrm{~ms}$. The ME-fMRI sequence was implemented using vendor EPI excitation and a modified EPI readout, and used on-line reconstruction (Poser et al., 2006). Each TR corresponded with the acquisition of three volumes having TE values of 15,35 , and $58 \mathrm{~ms}$.

Anatomical and functional imaging processing. Anatomical images were first processed using the FreeSurfer pipeline for skull stripping, segmentation, and cortical surface mesh construction. Separately, functional images were processed using the ME-ICA pipeline as implemented in the AFNI meica.py toolbox. This toolbox implements preprocessing, decomposition, and denoising steps for multiecho EPI data, which are detailed in the study by Kundu et al. (2015). Briefly, multiecho EPI time series datasets of each TE were aligned for slice-timing offsets, all volumes were aligned with rigid-body motion correction to the volume of the first $\mathrm{TR}$, and functional images were skull stripped. These processed functional images were used to compute $\mathrm{T}_{2}^{\star}$ and $\mathrm{S}_{0}$ maps by fitting signal means of different $\mathrm{TEs}$ for each voxel to a monoexponential decay model. Using meica.py, parameters of affine coregistration between (skullstripped) anatomical and functional images were estimated, using the thresholded $\mathrm{T}_{2}^{*}$ map as a weight volume (Kundu et al., 2015). Anatomical images were nonlinearly warped to MNI standard space using the AFNI 3dQwarp technique (Cox, 2012), then a single warp combining deobliquing, motion correction, anatomical-functional coregistration, and nonlinear warp to MNI space was applied to each echo dataset separately. This procedure produced the preprocessed datasets for input into decomposition and denoising analyses.

\section{Statistical analysis}

$B O L D$ components. After preprocessing, the next stage of ME-ICA was to extract BOLD components. The processing steps of the ME-ICA pipeline are summarized here and have been detailed in prior publications (Kundu et al., 2015, 2017). The first step of ME-ICA was to compute a weighted average of the separate echo time series into a single optimized time series dataset. The weighting function was evaluated at each voxel, factoring in $\mathrm{TE}$ and voxel-specific estimates of $\mathrm{T}_{2}^{\star}$ (Posse et al., 1999). The second step was to estimate the total number of components and to remove Gaussian distributed components from the data. This step is standard for all applications of ICA to fMRI data, and is usually performed using a variant of principal components analysis (PCA). The ME-ICA pipeline performs this step using ME-PCA (Kundu et al., 2013). The third step was to conduct the ICA on the resulting data and elucidate functional BOLD and artifact components (Kundu et al., 2012). Finally, the ICA components were organized into BOLD and non-BOLD categories using metrics of TE dependence and TE independence. Strong BOLD weighting of a component was expressed in high values of the pseudo- $F$ statistic $\kappa$, and strong non-BOLD (artifact) weighting was expressed in high values of the pseudo- $F$ statistic $\rho$. Functional BOLD components were interpreted as those with high $\kappa$ values and low $\rho$ values (Kundu et al., 2012, 2017). All remaining components are considered to be non-BOLD components. This component classification is also used to denoise fMRI time series. The non-BOLD components are projected out of the time series data, based on multiple least-squares fit of the entire mixing matrix. Maps of the signal-to-noise ratio (SNR) of these time series data were computed for each subject by dividing the voxel means by the standard deviation (SD).

Global age-related variation of BOLD components. The total number of BOLD components per subject was compared against subject age. The resting-state fMRI dataset from each subject produced one such measure. Based on prior reports of structural brain changes with age (Giedd et al., 1999; Paus et al., 2001), the relationship between component number and age was computed using linear, quadratic, exponential, and power law functions.

The validity of values of BOLD dimensionality derived from ME-ICA was further evaluated. First, BOLD dimensionality values were correlated against values of framewise displacement derived from rigid-body head motion parameters. This was done to determine whether BOLD dimen- 
sionality was significantly influenced by head motion. Second, BOLD and non-BOLD dimensionality values (determined simultaneously in ME-ICA) were correlated against each other. This was done to assess whether greater non-BOLD (i.e., artifact) signal variance predetermined the number of BOLD components. Last, ME-ICA-denoised BOLD time series were analyzed with probabilistic PCA (PPCA) from the FSL program MELODIC. This give an independent estimate of dimensionality for comparison with ME-ICA estimates of BOLD dimensionality.

We sought further to establish BOLD dimensionality as a metric of integration of functional brain activity, via comparison to corresponding graph theoretic measures based on functional connectomes. First, for each subject, functional connectomes were constructed. Each subject's Deskian-Killany FreeSurfer brain parcellation was coregistered to their preprocessed ME-ICA-derived BOLD functional time series. Parcel average time series were then computed, and used to compute a matrix of Pearson correlations. These matrices were then thresholded. Several thresholds were sampled: $r=0.3-0.9$, in $\Delta r=0.1$ increments. Negative correlations were removed, so only positive correlations were considered. Thresholded time series correlation matrices were then Fisher R-Z transformed to create functional connectomes. Graph theoretic analysis of connectomes was performed using the Brain Connectivity Toolbox, implemented in MATLAB (Rubinov and Sporns, 2010). For each subject connectome (weighted, undirected), Louvain community detection was applied. Per-node participation coefficient values were then computed based on those communities. The mean participation coefficient was used as a per-subject measure of functional connectomic integration. These per-subject values of mean participation coefficient were then compared against BOLD dimensionality using Pearson (parametric) and Spearman (nonparametric) correlations. This procedure was repeated for each sampled threshold on time series correlation to check for robustness of the comparison.

Regional age-related variation of BOLD components. The BOLD components identified using ME-ICA were used to delineate functional regions. A region was defined as a set of contiguous voxels with a common set of overlapping BOLD components. The effect of a component on a region was determined by the significance of BOLD TE dependence across the contiguous voxels in the component map. Component maps were rendered using the F- $\mathrm{R}_{2}^{*}$ statistic (Kundu et al., 2012). This statistic indicates the level of TE dependence [i.e., the susceptibility-weighted transverse relaxation rate $\left(R_{2}^{*}\right)$, the inverse of $\left.T_{2}^{*}\right]$. F- $R_{2}^{*}$ is computed voxelwise and follows a standard $F_{(1,2)}$ distribution. BOLD components (i.e., functional networks) were each rendered in $\mathrm{F}-\mathrm{R}_{2}^{*}$ units, thresholded ( $p<0.01$, uncorrected, as per prior work on statistical significance for TE dependence maps, Kundu et al., 2012), and binarized. Binary maps of all BOLD components were summed, producing a map of the number of components with significant weight at each voxel, in effect serving as a map of component overlap. Next, the relationship of how the number of overlapping BOLD components changed with age was mapped. A functional brain map showing the number of overlapping BOLD components per voxel was rendered for each subject, called a $\mathrm{T}_{2}^{*}$ component overlap map ( $\mathrm{T}_{2}^{*}$-COLAP). This map was normalized to standard cortical surface space using FreeSurfer cortical surface meshes and AFNI SUMA tools (Argall et al., 2006; Fischl, 2012), with subcortical regions from nonlinear warps merged to create a whole-brain standard space map.

Across subjects, for each voxel in the $\mathrm{T}_{2}^{*}$-COLAP maps in standard space, we computed a nonparametric Spearman correlation of the number of overlapping BOLD components versus subject age. This produced a new map reflecting Spearman correlation values, which was thresholded to Spearman's $\rho$ for $p<0.01$ significance, as shown in Figure $4 B$. The resulting parametric map was then cluster corrected, based on Monte Carlo $\alpha$ probability simulations, to $\alpha<0.05$. First, the smoothness of preprocessed multiecho functional data was estimated in terms of spatial autocorrelation using AFNI 3dFWHMx (with the -ACF option). Then, the cluster probability table for nearest-neighbor clustering (considering both positive and negative values) was calculated using $3 \mathrm{dCl}$ (ustSim (Cox et al., 2017). Finally, spatial clustering was applied using AFNI 3 dmerge. For the target cluster probabilities, a cluster extent of 38 voxels was required.

A separate spatial-clustering strategy for regional BOLD dimensionality correlation maps, called density-based spatial clustering of applica- tions with noise (DBSCAN; Ester et al., 1996), was also evaluated. This technique, a standard multivariate clustering technique, can deterministically cluster arbitrary feature spaces. Here we used DBSCAN on a feature space including both spatial coordinates (i.e., spatial clustering) as well as a data variate of the Spearman correlation values, as described above. DBSCAN formally distinguishes clusters and noise, defining clusters on density and noise in terms of being outside of "density reachability." Practically, this application of DBSCAN is well suited to spatially clustering a densely populated statistical parametric map while rejecting voxels with neither high value nor contiguity with larger clusters. In such cases, otherwise distinct clusters tend to have some touching voxels, yielding apparent contiguity and thus very large clusters. Instead of applying an arbitrary refinement scheme like erosion and dilation after spatial clustering, we chose DBSCAN as an optimization-based clustering technique to solve the spatial contiguity problem. The Python scikitlearn implementation of DBSCAN was used. A parameter search for the single DBSCAN parameter $\eta$ was conducted, based on a minimum cluster size of 40 to find the solution that yielded the maximum number of clusters. Each surviving cluster was then treated as a separate mask. For each subject map, component overlap values were averaged within each mask, and values were correlated against age for each mask region. The relationships between average regional BOLD component overlap and subject age was assessed for linear, exponential, and power law relationships.

Cross-validation of age as predicted by regional BOLD component overlap. The extent to which patterns of regional BOLD component overlap were predictive of age was assessed using support vector machine (Scholkopf et al., 1997), cross-validation, and permutation testing, implemented in Python software (Pedregosa et al., 2011). The BOLD component overlap values from all voxels of $\mathrm{T}_{2}^{\star}$-COLAP maps across subjects were used as features for age prediction. Prediction was made using the support vector regression (SVR) framework, training on values of age. We confirmed that raw BOLD component counts gave inferior prediction compared with log-linearized counts, so the latter is shown in the results. The accuracy of the age prediction from BOLD component overlap was determined using leave-one-out (LOO) cross-validation. The mean and standard deviation of the prediction error were computed. The significance of classification was determined by permutation testing. Using 1000 randomly permutated assignments of ages to datasets, the SVR was trained and tested in $\mathrm{LOO}$ cross-validation, with median absolute error as the loss function. In effect, the significance of association between "true" age and BOLD dimensionality was established by comparison to chance association between an "age-like" variate and dimensionality values, and was expressed in a significance $(p)$ value.

Seed-based functional connectivity of regions with age-related change in $B O L D$ components. Functional connectivity analysis was conducted based on time series extracted from those brain regions that showed a significant change in BOLD component overlap with age. For each subject, voxel time series within each respective cluster mask were averaged. The Pearson correlation between these time series was computed, and was scaled to correct for the effective degrees of freedom for correlation (i.e., the number of BOLD independent components comprising the denoised time series) according to the following (Kundu et al., 2013):

$$
\mathrm{Z}=\operatorname{arctanh} * \operatorname{sqrt}(\mathrm{N}-3) .
$$

For each region, the Fisher $Z$ map of functional connectivity for each subject was input to a one-sample $t$ test group analysis, producing a group map of functional connectivity for that region. Each such group map was thresholded with cluster correction for multiple comparisons based on $\alpha$ probability simulation (as described above) to $\alpha<0.01$ (Cox et al., 2017). Then, the overlap of each thresholded group-level regional connectivity map was computed as a count of voxels. These corrected maps were used to determine which regions had seed-connectivity maps that overlapped with seed-connectivity maps of the other regions, which was represented in a graph.

Covariation of functional connectivity with age. We tested the relationship between regional changes in component overlap versus functional connectivity and its change with age. The clusters of the map of Spearman correlation between $\mathrm{T}_{2}^{\star}$-COLAP maps and subject age were used to 
A

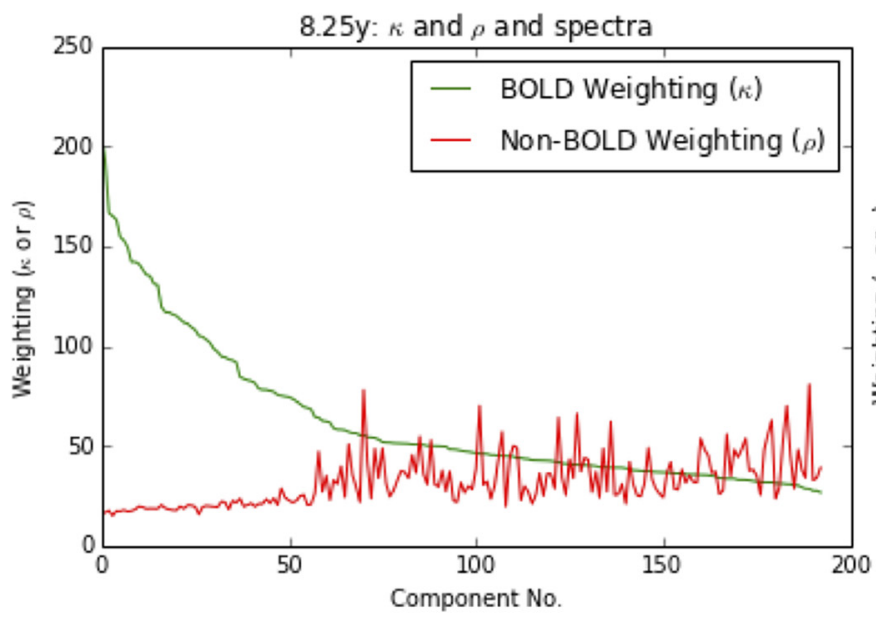

C

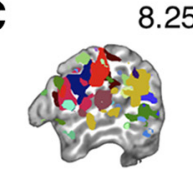

8.25 y Component overlap ( $\mathrm{F}_{\mathrm{R} 2^{*}}$ maps)
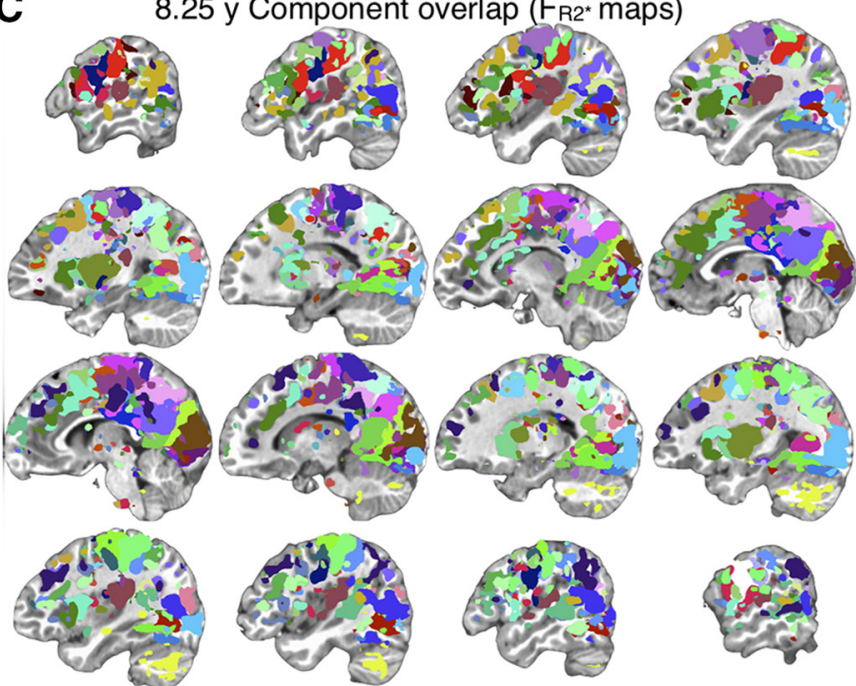

D

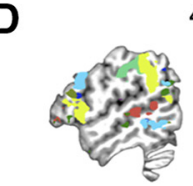

46.17 y Component overlap ( $\mathrm{F}_{\mathrm{R} 2^{\star}}$ maps)
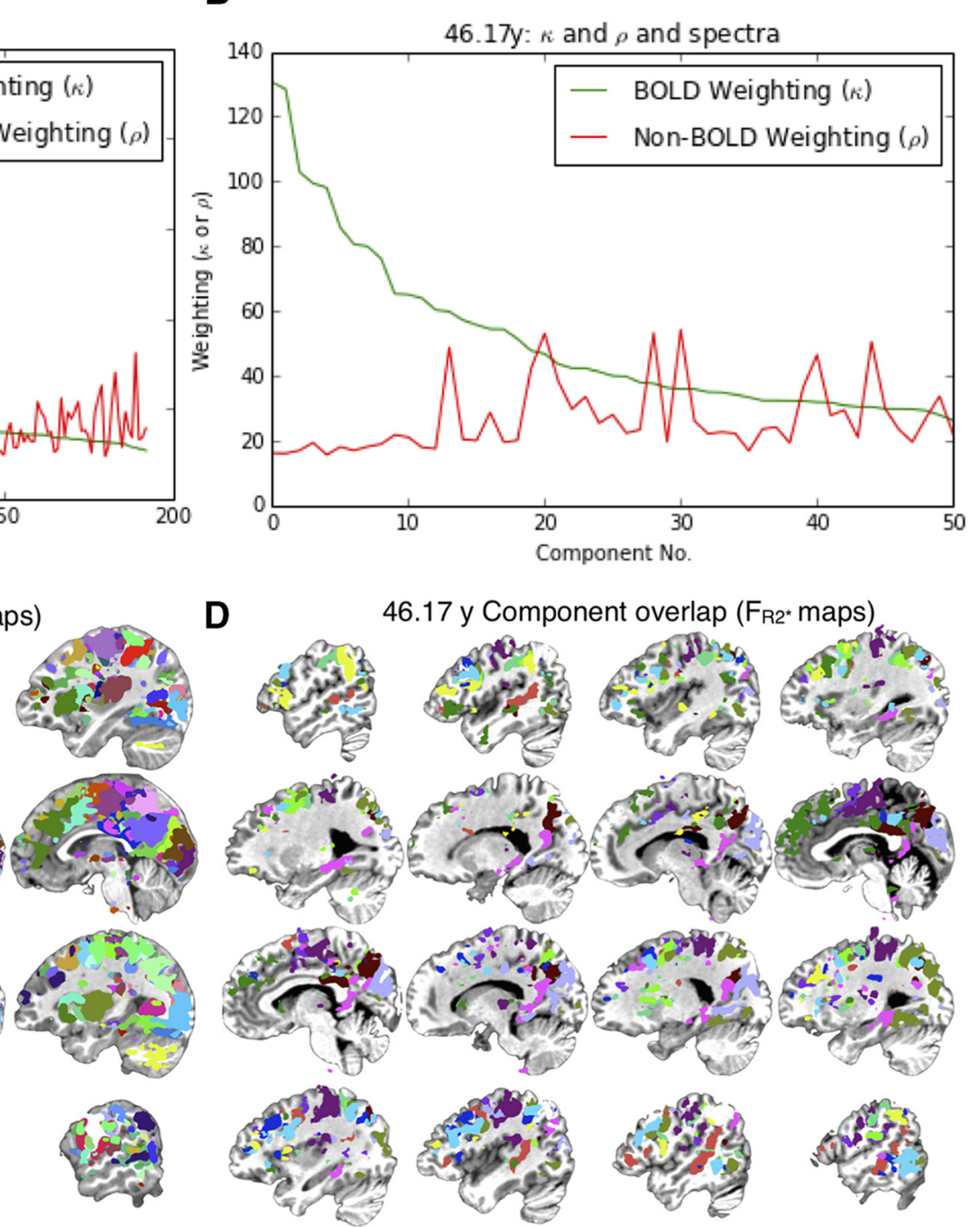

Figure 1. $\quad \boldsymbol{A}, \boldsymbol{B}, \boldsymbol{\kappa}$ and $\rho$ values (i.e., pseudo-F statistics) for component-level TE dependence and TE independence of ME-ICAs for youngest $(\boldsymbol{A})$ and oldest $(\boldsymbol{B})$ subjects expressed as $S c r e e$ plots, sorted by $\kappa$ values. Functional network components are characterized by high $\kappa$ and low $\rho$ values. Note the transition from the $\kappa \gg \rho(B D L D)$ regime to the $\kappa \leq \rho($ non-BOLD) regime. $C, D, O v e r l a p$ of BOLD components (thresholded $\alpha<0.01$ ) from subjects of the youngest and oldest ages, respectively ( 8.25 and 46.17 years of age). The colored regions are derived from thresholded BOLD components. These component maps are overlaid in order of decreasing component size (in terms of number of significant voxels). C, Overlap of 53 BOLD components. D, Overlap of 13 BOLD components.

identify centroids to use as seed voxels for functional connectivity analysis. The Pearson correlations of each such time course against the time courses of all other voxels within the brain mask were computed, yielding whole-brain connectivity maps. Time series correlation values were normalized using the Fisher $R-Z$ transform. The standard error (SE) term that accounted for the variability across datasets in terms of the total number of BOLD components (Eq. 1) was included, because falsepositive error may be introduced when this factor is not accounted for (Kundu et al., 2013). Group analyses of these connectivity maps were then performed using mass-univariate Pearson correlation analysis of connectivity versus age, voxelwise. For each seed, the number of voxels with significant $(p<0.01)$ group-level correlation of connectivity with age was counted, separately for positive and negative values. A twosample paired $t$ test was then used to compare the number of positively versus negatively age-correlated voxels, to determine whether there was a net increase or decrease of functional connectivity with age across regions that showed decreasing regional BOLD dimensionality.

\section{Results}

ME-ICA of resting-state fMRI across adolescent and adult data

The separation of ME-ICA components into BOLD and nonBOLD categories was conducted successfully for each subject. $\kappa$ and $\rho$ metrics for datasets of a representative subsample of subjects between 8.25 and 46.17 years are shown in Figure 1, $A$ and $B$, respectively. $\kappa$ metrics are plotted in Scree plots in descending order, juxtaposed with corresponding $\rho$ values (i.e., in the order of $\kappa$ values). Together, a change is apparent from high- $\kappa /$ low $-\rho$ components to low- $\kappa /$ high- $\rho$ components. These two categories represent the two distinct BOLD and non-BOLD sets of components, from which respective total component numbers were determined. Figure 1 shows for, an adolescent and an adult subject, maps of the "projections" of all components into a single map, in terms of the most statistically significant clusters (Fig. 1C,D). On visual inspection of these maps, the adolescent subject dataset shows a wider coverage of gray matter by nodes of detected functional networks, including in subcortex and cerebellum.

Global BOLD component number decreases with age

Global BOLD component numbers decreased with age in all tested relations, as follows: power law, exponential, and linear $\left(R^{2}=0.32,0.33,0.28\right.$, respectively). Agreement with all three relations is consistent in the present case as they were all mono- 
A

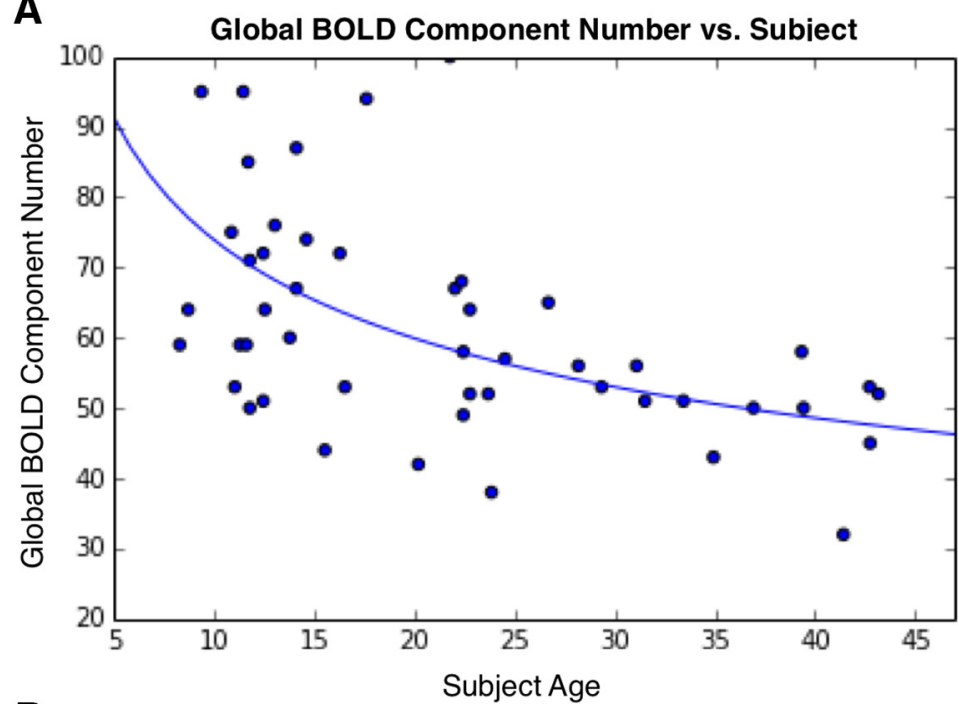

B

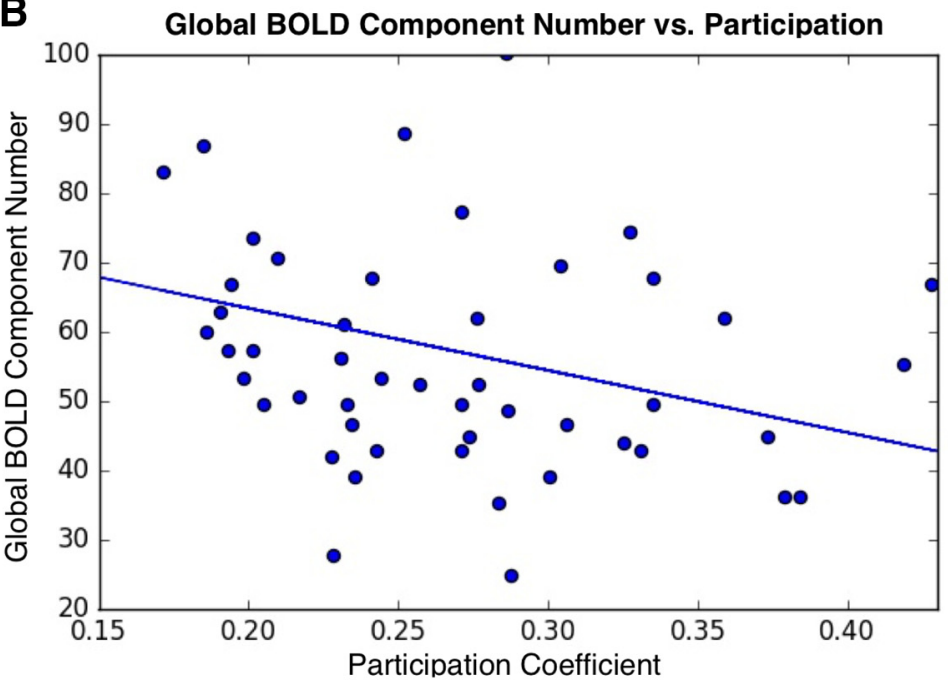

Figure 2. A, Scatterplot of global BOLD component numbers across subjects. Power law fit (blue) is shown for the scaling of component number versus subject age. Significant linear $(\mathrm{L})$ and power law $(\mathrm{PL})$ trends were found $\left(\mathrm{L}: R^{2}=0.29, p<0.001 ; \mathrm{PL}\right.$ : $\left.R^{2}=0.30, p<0.001\right) . B$, Scatterplot of global BOLD component number versus corresponding subject values of mean participation coefficient, based on functional connectomes of the Deskian-Killany parcellation and Louvain community detection. mean participation coefficient based on thresholded, weighted, undirected functional connectomes was significantly $(p<$ $0.05)$ correlated with global BOLD dimensionality. The connectivity threshold that led to the most significant such correlation was $R>0.5$, leading to correlations between BOLD dimensionality and participation coefficient of Pearson's $r=-0.34(p=0.013)$ and Spearman's $\rho=-0.38(p=0.0057)$.

\section{Regional BOLD component count and its reduction with age}

Maps of BOLD components were generated in units of TE dependence $\left(\mathrm{F}-\mathrm{R}_{2}^{*}\right.$; thresholded at $\alpha<0.01$, corrected). The mapping of the overlap of BOLD components, the $\mathrm{T}_{2}^{*}$-COLAP map, is shown for a representative subject in Figure $4 A$. The TE dependence maps of ICA components on which the $\mathrm{T}_{2}^{*}$-COLAP map is based show spatial patterns comparable with conventional amplitude-based maps from spatial ICA. The components mapped with TE dependence as found in individual subject data included canonical restingstate networks such as default mode, sensorimotor, and frontoparietal networks (Damoiseaux et al., 2006).

For a representative subject, Figure $4 A$ illustrates the result of thresholding, binarizing, and summing over BOLD component maps to produce a component overlap map. The overlap map conveys the number of BOLD components with a significant linear TE dependence at each voxel. For example, high component overlap is observed for regions associated with the default-mode network, and low component overlap is observed in regions such as the motor cortex.

tonic, and the effect size was large. The maximum global BOLD component number was seen for a 9-year-old subject, who expressed 95 BOLD networks. The minimum value was for a $42-$ year-old subject, who expressed 32 BOLD networks. Figure 2 shows associations of subject age with global BOLD component number. In addition, we confirmed that global BOLD component number was not correlated to the level of subject head motion. The number of BOLD components was also not correlated to the number of non-BOLD components $(r=0.04, p=0.7)$. In contrast, the level of subject head motion was correlated to the number of non-BOLD components according to a positive linear relationship, which is shown in Figure 3.

We compared the estimate of BOLD dimensionality from ME-ICA to a separate estimate of dimensionality provided by PPCA of the time series with non-BOLD signals removed. We found a highly significant correlation between the two dimensionality estimates $\left(r=0.97, p \ll 10^{-7}\right)$. BOLD dimensionality from ME-ICA also correlated with graph theoretic measures of integration derived from subject-level functional connectomes. Across connectivity thresholds corresponding to $r=0.4-0.8$, the
TE dependence maps and their overlap showed two additional aspects of component maps of functional networks. One is that functional networks, rendered in units of TE dependence, are typically inclusive of widespread brain regions. This is attributed to lowamplitude contributions of components being detected in TE dependence maps that may not have been detected in maps in magnitude units thresholded according to $Z$-scores. Second is the indication that TE dependence maps of networks can be more comprehensive than magnitude-based maps on the basis of greater overlap across components, which is not seen in the case of magnitude-based maps.

\section{Regional BOLD component reduction with age}

The relationship between the number of overlapping BOLD components and subject age at group level was mapped for each voxel using a Spearman correlation. After voxel and cluster thresholds were applied to this correlation map (see Materials and Methods), a distinct set of clusters was found. These clusters indicated an age-related decrease in BOLD component count in gray matter regions. The cluster averages of regional BOLD component over- 
A
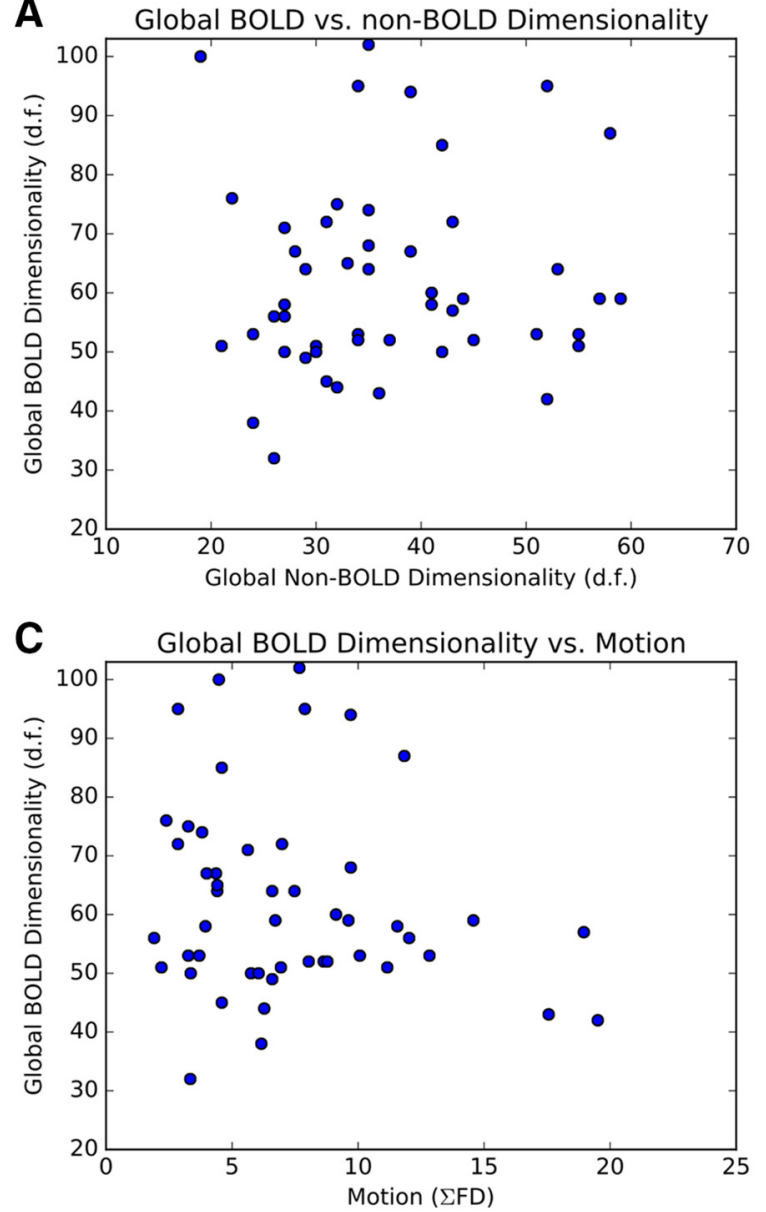

B
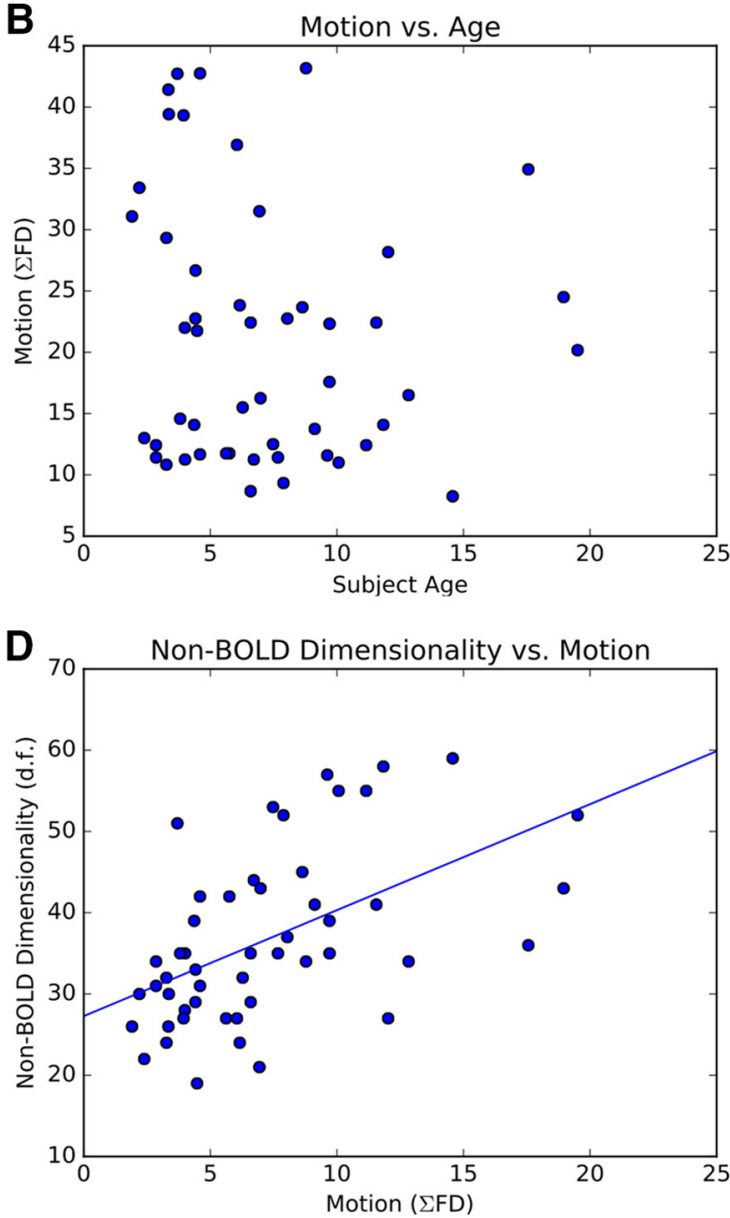

Figure 3. Scatterplots of non-BOLD component number across subjects showing significant linear ( $L$ ) or power law (PL) fits. $A$, Total $B O L D$ component number versus total non-BOLD component number, no significant relation $(r=0.05, p=0.7) \cdot \boldsymbol{B}$, Global BOLD component number versus subject motion, no significant fits $\left(\mathrm{L}: R^{2}=0.02, p=0.26 ; \mathrm{PL}: R^{2}=0.02, p=0.24\right) . C$, Subject motion versus age, no significant relation (L: $\left.R^{2}=0.02, p=0.31 ; \mathrm{PL}: R^{2}=0.01, p=0.58\right) . D$, Global non-BOLD dimensionality versus subject motion, significant linear relationship $\left(\mathrm{L}: R^{2}=0.30, p=\right.$ $\left.0.001 ; \mathrm{PL}: R^{2}=0.30, p=0.001\right)$.

lap versus age showed highly significant decreases of BOLD component count with age across regions (Fig. 4B). Thus, at both voxel and cluster levels, age-related decreases in component count were observed. Importantly, the reduction of component count was not homogeneous across the brain. Figure $4 B$ maps specific regions that showed this reduction, averaged over the voxels of the region, as follows: prefrontal cortex (i.e., bilateral dorsolateral prefrontal cortex, medial prefrontal cortex, frontopolar cortex), bilateral superior parietal cortex (i.e., sensory cortex, precuneus), and bilateral cerebellar hemispheres spanning Crus VI-VIIab. Regions that did not show age-related change in BOLD component overlap included premotor and primary motor cortices, the temporal lobes, insula, thalamus, and inferior frontal cortex.

Linear, exponential, and power law models were used to characterize regional age-related reduction of BOLD component overlap with age. The power law model gave the most statistically significant fit $(r=-0.54, p \ll 0.001)$. The finding of regional reduction in BOLD component overlap was consistent with the global reduction of BOLD component number, but with even greater statistical significance at the regional level than the global level.

Cross-validation of subject age as predicted by regional variation of BOLD component overlap

Regional values of BOLD component overlap were used to predict subject age using a SVR and cross-validation strategy (Fig. 5). This approach predicted age with an average error of $6.5 \pm 0.6$ years, with error based on leave-one-out cross-validation. The significance of the classification, based on cross-validation involving 1000 permutations of age values, was $p=0.009$. These results suggest that regional BOLD component overlap is a significant predictor of subject age on the order of years.

Seed-based functional connectivity between regional changes in BOLD component overlap

Seed-based connectivity was computed between functional regions that showed age-related changes in the number of overlapping BOLD components. Seed-based connectivity was computed using the complete Fisher transformation including degrees of freedom in terms of the global BOLD component number, a subjectlevel variable. The group-level connectivity between these regions was represented in a circular network diagram (Fig. 6). Each region of this network showed connectivity to at least one of the other regions with age-related change in component overlap. Bilateral cerebellum and right precuneus were most connected to the other regions (i.e., these were the highest degree nodes).

Correlation of seed connectivity with age after controlling for total BOLD components

We found that the regions with decreasing regional BOLD dimensionality with age had increasing functional connectivity with age. 

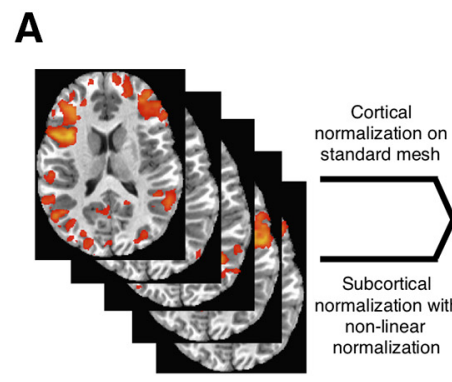

B
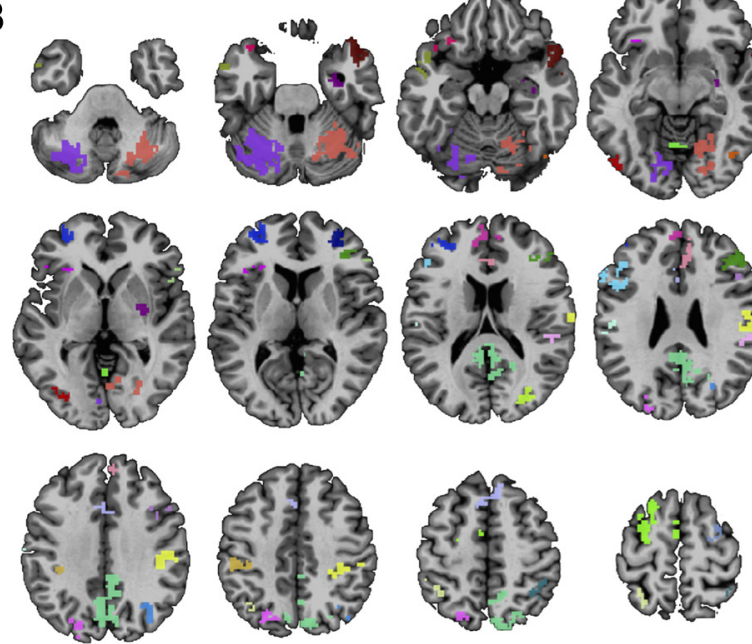

C
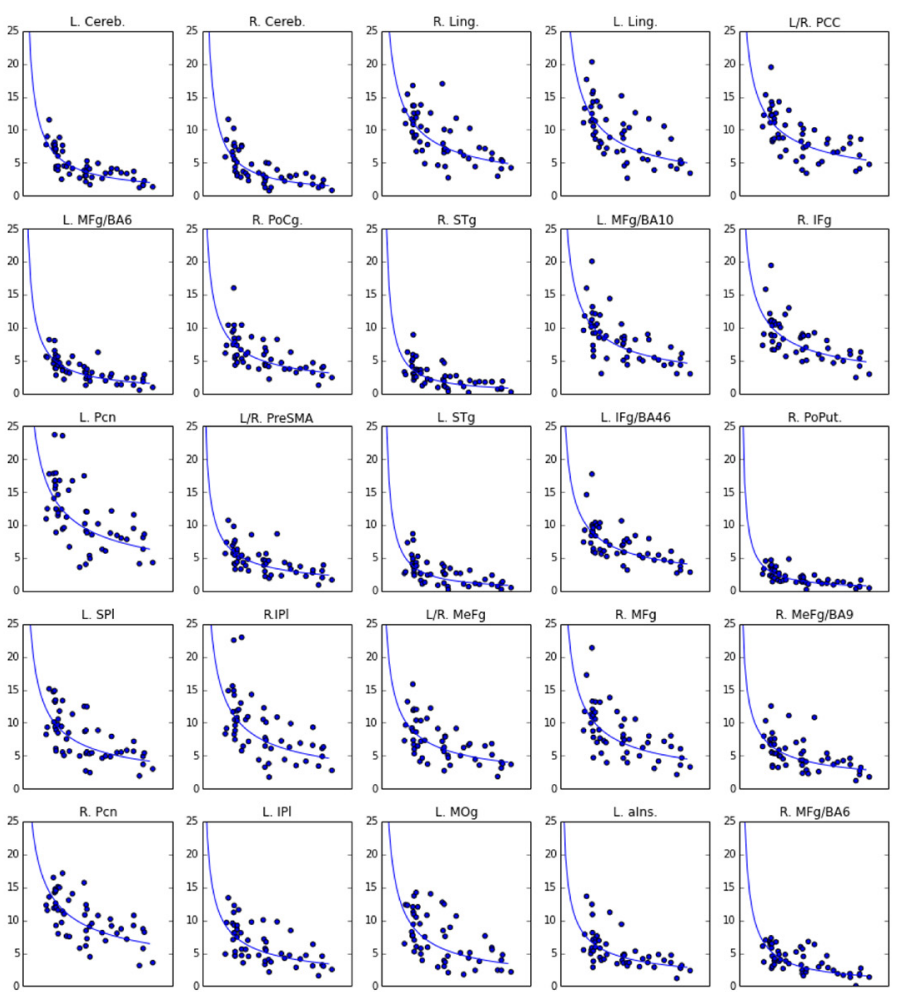

Figure 4. Assessment of regional BOLD component overlap versus subject age. $\boldsymbol{A}$, Procedure: thresholded ( $p<0.01$, uncorrected) $F$-maps indicating component voxels with significant TE dependence are warped into standard (MNI) space via normalized cortical surface mesh and nonlinear volumetric warp (see Materials and Methods), then are binarized and summed to produce an overlap map for component-level TE dependence indicating regional BOLD dimensionality. These were termed T2-COLAP maps. B, Voxels (grouped into clusters post hoc) showing significant Spearman correlation of component overlap with subject age. Clusters represent significance with correction for multiple comparisons $(\alpha<0.01)$, rendered with a high-contrast color map. C, Scatterplots representing cluster averages for regional BOLD component overlap plotted against subject age. A high magnitude of power law behavior is apparent (note that the initial voxelwise correlation was nonparametric), which varies in parameters depending on brain region. BA, Brodmann's area; (ereb., cerebellum; L., left; R., right; PreSMA, pre-supplementary motor area; Ling., lingual gyrus; PCC, posterior cingulate cortex; MFg, middle frontal gyrus; PoCg., posterior cingulate gyrus; STg, superior temporal gyrus; IFg, inferior frontal gyrus; Pcn, precuneus; PoPut, posterior putamen; SPI, superior parietal lobule; IPI, inferior parietal lobule; MeFg, medial frontal gyrus; MOg, middle occipital gyrus; alns, anterior insula.

Across these regions, we found that the number of voxels with significant trends of increasing functional connectivity with age were significantly greater $(p=0.0004)$ than voxels with decreasing trends of connectivity with age (Fig. 7A). The two-sample $t$ test design of this analysis was robust to false-positive error. The right cerebellum showed particularly high change of functional connectivity with age. The analysis of seed-based functional connectivity of the cerebellum versus subject age is shown in Figure 7 , $B$ and $C$. Age-related connectivity was seen from the cerebellum to a bilateral frontoparietal network, caudate head, and medial thalamus (Fig. $7 B$ ). Age-related connectivity increases were greater in the right hemisphere. Cluster averages of the values of connectivity across subjects versus subject age are shown in Figure 7C. Except for the right parietal lobe, which showed decreasing connectivity with age, all plots showed significant age-related increases in connectivity with age, even after controlling for global age-related change in BOLD component number.

\section{Discussion}

In this study, we showed that age modulates the number of components of functional brain activity in the resting state. This association suggests increasing age-related integration of functional brain networks, the first such demonstration in restingstate fMRI data. The number of BOLD components varies with age parametrically, as a power law. This pattern is highly robust. It is expressed with high statistical significance at the global whole-brain level, and with even greater significance locally, in individual brain regions, expressed as a change in the number of overlapping BOLD components as a function of age. The effect is not expressed across all brain regions homogeneously. Frontoparietal and sensory association cortices show the regionally specific effect, while other regions do not. The ME-fMRI approach allowed us to take a data-driven approach to finding those brain regions that showed age-related changes. The estimation of seed-based connectivity among these regions, after controlling for the effect of the number of BOLD components, showed that these regions form a functional network with each other. Among regions with age-related change in BOLD component overlap, the cerebellum was found to be the most highly connected to the others, as well as to have the greatest change in connectivity with age.

The pattern of change in component number versus age is consistent with expectations based on age-related structural brain change. Brain development is a complex process that continues into early adulthood. After a fourfold increase in brain volume from birth to about 5 years of age, gross brain morphology stabilizes, with only an $\sim 10 \%$ increase in total volume up to adulthood. In adulthood, up to the third decade of life, microstructural change is the main mechanism of brain development (Raz, 2004; Lebel et al., 2008). Microstructural changes include increasing myelination of white matter and synaptic pruning. The inter-relationship of brain structure and function leads to an expectation that the functional 
organization of the brain would also change substantially due to brain development. In this study, we show evidence of such change in terms of functional organization as well as a key statistical characteristic of functional organization called BOLD dimensionality, or component count. Recent work has already shown that network segregation is one of the most robust and reproducible findings in developmental network neuroscience (Fair et al., 2007; Supekar et al., 2009; Dosenbach et al., 2010; Baum et al., 2017). Studies have consistently shown that short-distance connectivity decreases while long-distance connectivity increases with subject age. This pattern in fact concurs with the decrease in BOLD dimensionality with age, as more regionally specific networks that dominate at younger ages integrate into anatomically distributed and functionally distinct networks over neurodevelopment. The present work specially demonstrates that this pattern of the integration in the developing connectome also manifests with a change in the global and regional statistical characteristics of BOLD signal itself.

Virtually all fMRI studies on functional connectivity to date are based on the assumption that the number of signal components in restingstate data is a random effect (van den Heuvel and Hulshoff Pol, 2010). This is evidenced by the current standard of estimating connectivity based on a version of the Fisher transform that drops its SE term. This term would normally be used to control for dataset-level variability in the number of components in the time series data overall. In a prior study, we showed that this assumption is not valid from a methodological standpoint. Specifically, head motion reduces the number of BOLD components by reducing acquisition sensitivity (Kundu et al., 2013). Here we provide the first evidence that the assumption is also invalid on a neurobiological basis because the number of signal components varies systematically with age.

It is a basic principle for how correlation is interpreted, from the canonical form of the Fisher R-Z transform, that an accurate estimate of the statistical significance of correlation depends on a proper factoring of the number of independent components in the data. Given the magnitude of the effect of age-related change in the number of BOLD independent components, up to a factor of three between adolescence and middle age, the consideration of BOLD component counts may be important for interpreting correlation-based connectivity estimates along the lifespan and in comparisons involving disease. The ongoing European Autism Interventions-Multicenter Study (EU-AIMS) study is acquiring multiecho resting-state fMRI in healthy individuals and patients with autism across the age range, and that study will permit further assessment of this effect in health and disease (Murphy and Spooren, 2012).

The present analysis incorporated subject age as a correlate of BOLD component number, which in turn led to the identification of brain regions with similar trajectories of functional change with age. These regions included dorsolateral and dorsomedial prefrontal cortex. The developmental sensitivity of these regions found here is consistent with existing findings of their prolonged developmental trajectory. The dorsal prefrontal cortex mediates the most complex, higher-level aspects of cognition (Petrides, 2000; Koechlin et al., 2003; Koechlin, 2011; Passingham and Wise, 2012). Activity within the dorsolateral prefrontal cortex relates mostly to monitoring and sequencing information in working memory (Petrides, 1995, 2005; Owen et al., 1998; Amiez and Petrides, 2007). It also relates to the hierarchical organization and sequencing of other cognitive operations and to performance monitoring (Duncan and Owen, 2000; Koechlin et al., 2003; Duncan, 2010). Activity in the dorsomedial prefrontal cortex is associated with social cognition both when making judgments about others' mental state or intentions (Amodio and Frith, 2006; Lieberman, 2007; Behrens et al., 2008; Krienen et al., 2010) and while reflecting on one's own self, beliefs, intentions, and actions (Amodio and Frith, 2006; Brass and Haggard, 2007; Desmet et al., 2011). Importantly, these functions are considered to reach maturity only in adulthood. This study also brings into focus age-related changes in the precuneus, which only recently has become prominent in the study of brain development (Dosenbach et al., 2010). The precuneus is a central node of the default-mode network, which is involved in self-referential processing. However, further work is needed to examine the behavioral significance of age-related regional variability in BOLD components.

The results also highlight a potentially critical role of the cerebellum in age-related brain connectivity. The high signal-tonoise ratio (SNR) of the cerebellum after ME-ICA denoising enabled the detection of substantial age-related changes in BOLD dimensionality and functional connectivity in this region. Historically, the cerebellum has been viewed mostly as a motor control region, but more recently there is increased recognition of its role in the regulation of autonomic function and cognition (Reis 


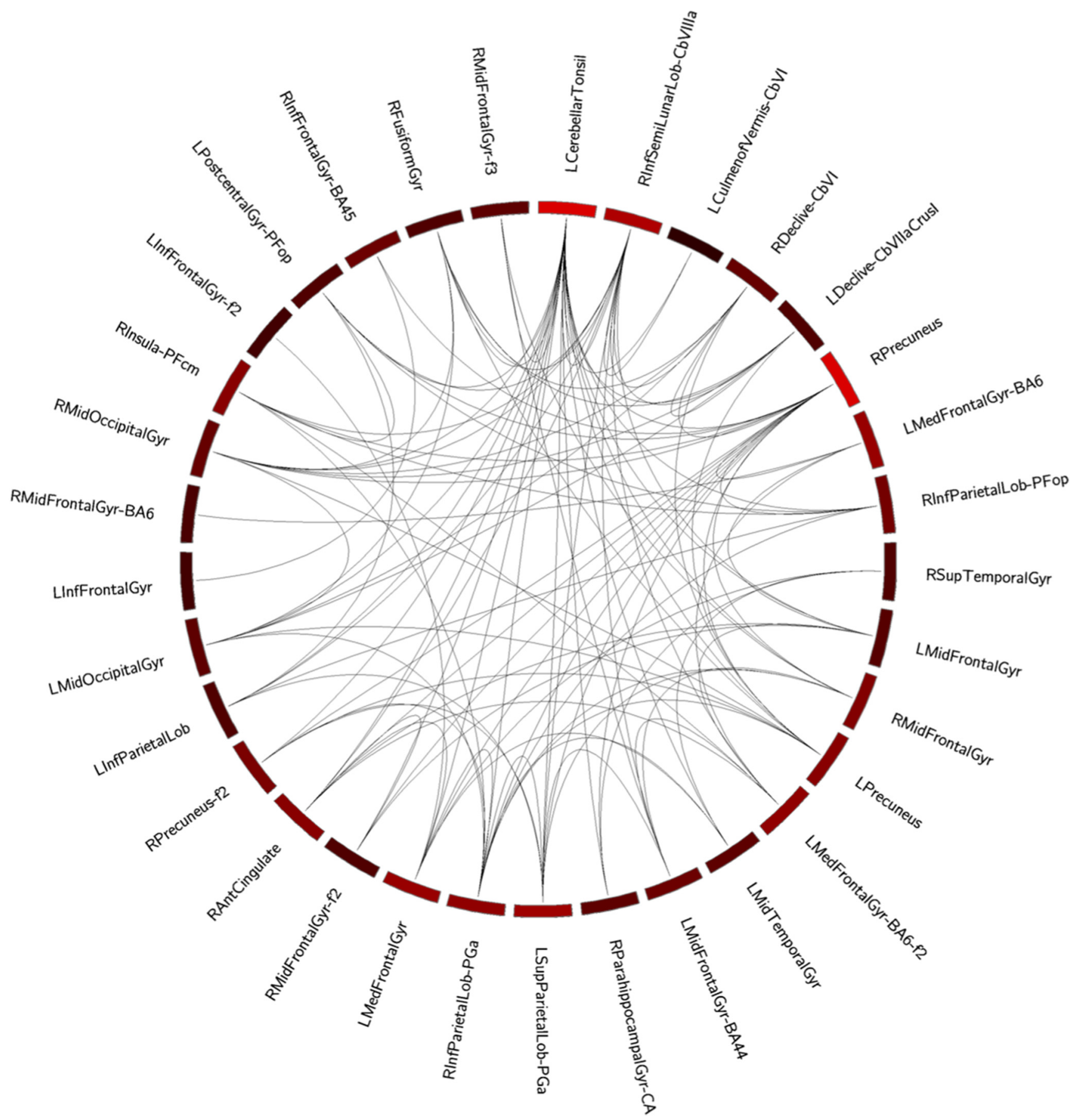

Figure 6. Circle plot representing the graph of average inter-regional connectivity between regions with decreasing BOLD component overlap versus subject age. Each edge that is shown represents positive connectivity of a seed and target region, such that connectivity is detected in the target region with cluster-level significance of $\alpha<0.01$. Self-connections are not shown. The degree of connectivity of each region is shown, with dark coloration representing a relatively low degree of connectivity, and bright red coloration representing a high degree of connectivity. A high degree of connectivity is observed of left and right cerebellar nodes as well as right precuneus. Inf, inferior; Sup, superior; Mid, middle; Gyr, gyrus; Ant, anterior; Med, medial; Lob, lobule; fi (e.g. f2, f3), focus i; Cb, cerebellar lobule; PFop, PFcm, PGa are cytoarchitectonic regions as defined in von Economo and Koskinas, 1926.

and Golanov, 1997; Parsons et al., 2000; Craig, 2002; Singer et al., 2004) and in affective processing (Schmahmann and Caplan, 2006). Cerebellar lesions give rise to a constellation of cognitive and affective abnormalities (i.e., the cerebellar cognitive affective syndrome; Schmahmann and Sherman, 1998). From a developmental standpoint, the cerebellum is reported to have low heritability in morphology, indicating a greater role of environment in its neurodevelopment (Gilmore et al., 2010). Functional neuroimaging studies report that regions of the cerebellar cortex most responsive to cognitive demands-namely lobules VI and VII (Stoodley and Schmahmann, 2009; Stoodley et al., 2012)interact closely with prefrontal and parietal association cortices (Habas et al., 2009; Buckner, 2013). These prior findings agree with the present results that show augmentation in the functional relationship between prefrontal regions and the cerebellum during brain maturation between adolescence and middle adulthood, suggesting the need for further study for this critical brain region (Raz et al., 1992). 
A

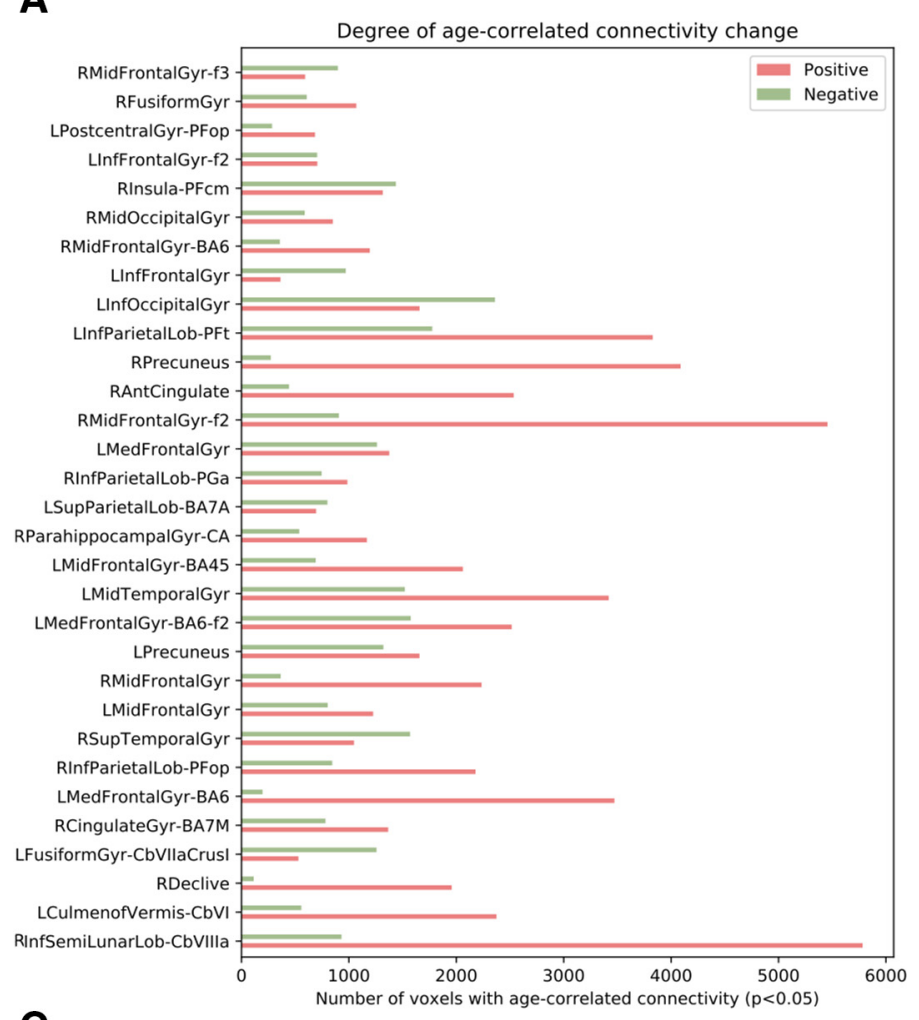

C
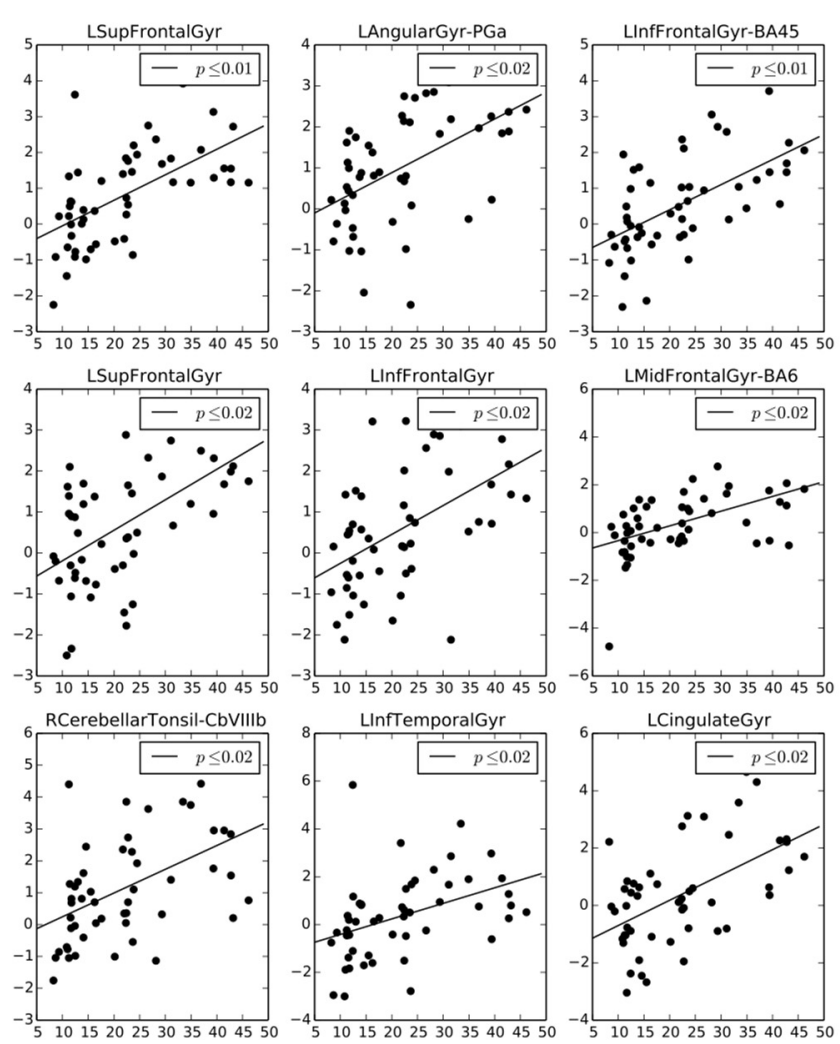

B

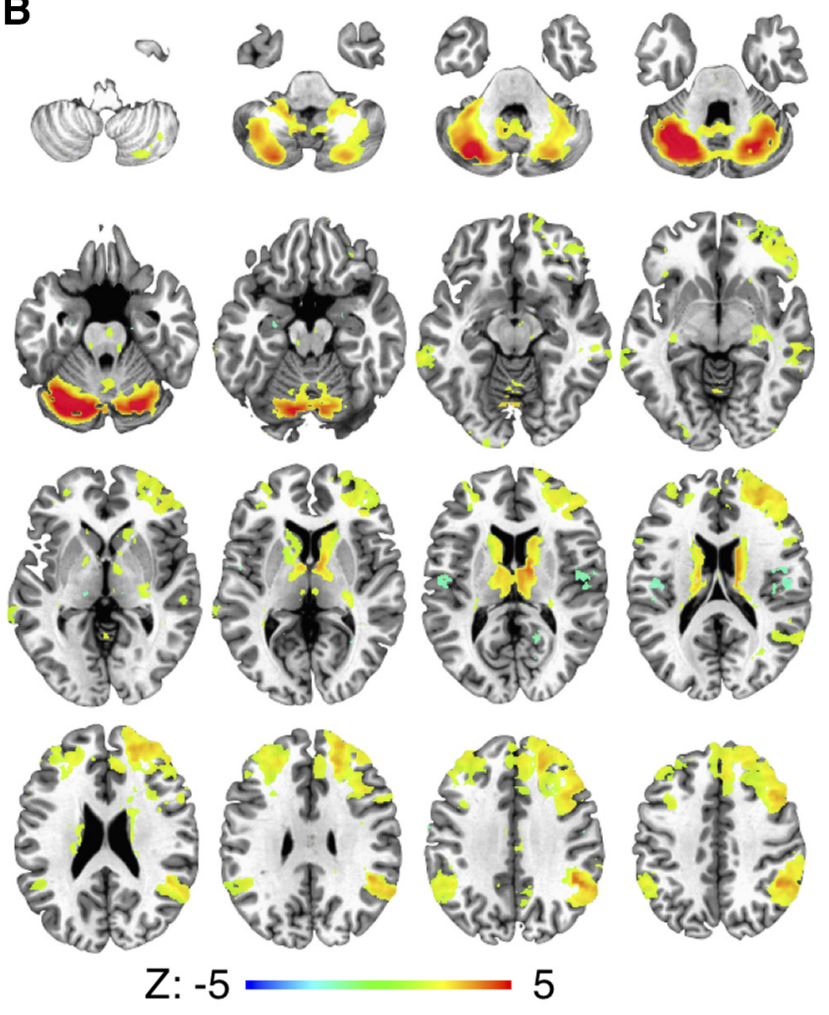

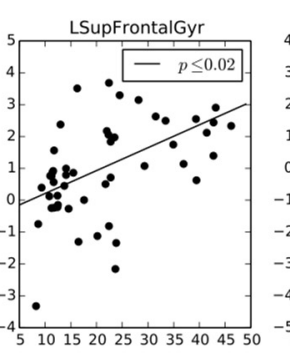
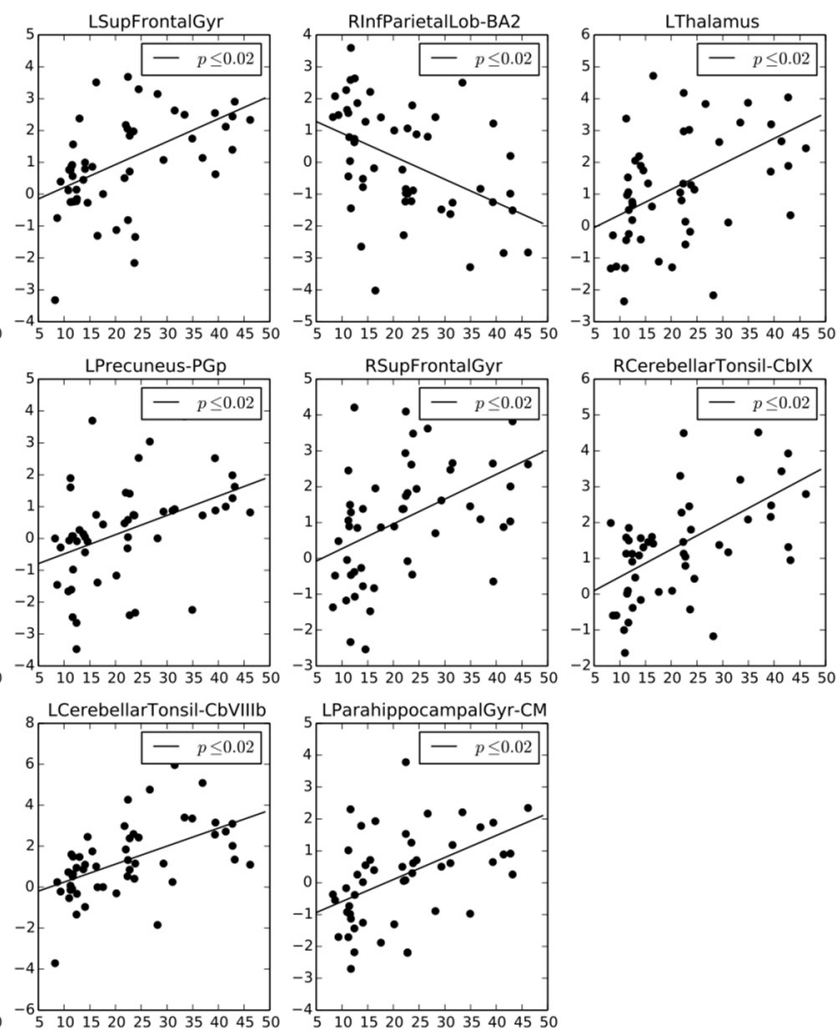

Figure 7. $A$, Bar charts comparing number of voxels with positive versus negative trends $(p<0.05)$ of change in seed-based functional connectivity with age, with seeds being those regions with changing BOLD component overlap with age. $\boldsymbol{B}$, Group-level seed-based functional connectivity map of left cerebellum (Lobule VII-Crus II), using central voxel of cerebellar cluster as seed and one-sample $t$ test, thresholded $\alpha<0.01$ (cluster corrected). Blue colors indicate mean negative functional connectivity. C, For left cerebellum seed connectivity, scatterplots showing mean connectivity per subject versus subject age, within clusters $(\alpha<0.01)$ showing age-correlated connectivity change. Linear fits are overlaid, with significance of the Pearson correlation in the legend. 
The limitations of the present study include a relatively small sample size compared with recent large-scale or multisite neurodevelopmental studies. Larger studies, especially those using MEfMRI, will be better suited to evaluate the relationship of BOLD component number and gender and cognitive variables, after factoring out the apparently large effect from subject age. To better understand the underlying neurobiological processes involved in the global and regional changes in BOLD component number, imaging of brain microstructure may be necessary. This would involve the additional acquisition of diffusion weighted imaging that is sensitive to processes like myelination or synaptic pruning, such as neurite orientation dispersion and distribution imaging (Zhang et al., 2012). To elucidate whether functional and microstructural changes also correlate to changes in brain metabolites, magnetic resonance spectroscopy (MRS) will be needed, possibly with a focus on regions that show change in BOLD component number. Also important is the consideration of how changes in BOLD component number relate to age-related changes in variability in regional activation during a task, which has already been associated with subject age (Garrett et al., 2011). Quantitative measurements of cerebral blood flow and metabolism alongside ME-ICA may also be informative as to how agerelated hemodynamics may relate to BOLD component number. This is possible given a recent MRI sequence that acquires multiecho multiband EPI with simultaneous arterial spin labeling, and has been shown to be compatible with ME-ICA (Cohen et al., 2017). Altogether, future studies could use advanced MRI sequences in imaging larger cohorts with in-depth behavioral data to better characterize the neurobiological changes that agerelated change in BOLD component number reflects.

\section{References}

Amiez C, Petrides M (2007) Selective involvement of the mid-dorsolateral prefrontal cortex in the coding of the serial order of visual stimuli in working memory. Proc Natl Acad Sci U S A 104:13786-13791. CrossRef Medline

Amodio DM, Frith CD (2006) Meeting of minds: the medial frontal cortex and social cognition. Nat Rev Neurosci 7:268-277. CrossRef Medline

Argall BD, Saad ZS, Beauchamp MS (2006) Simplified intersubject averaging on the cortical surface using SUMA. Hum Brain Mapp 27:14-27. CrossRef Medline

Baum GL, Ciric R, Roalf DR, Betzel RF, Moore TM, Shinohara RT, Kahn AE, Vandekar SN, Rupert PE, Quarmley M, Cook PA, Elliott MA, Ruparel K, Gur RE, Gur RC, Bassett DS, Satterthwaite TD (2017) Modular segregation of structural brain networks supports the development of executive function in youth. Curr Biol 27:1561-1572.e8. CrossRef Medline

Behrens TE, Hunt LT, Woolrich MW, Rushworth MF (2008) Associative learning of social value. Nature 456:245-249. CrossRef Medline

Brass M, Haggard P (2007) To do or not to do: the neural signature of self-control. J Neurosci 27:9141-9145. CrossRef Medline

Buckner RL (2013) The cerebellum and cognitive function: 25 years of insight from anatomy and neuroimaging. Neuron 80:807-815. CrossRef Medline

Cohen AD, Nencka AS, Lebel RM, Wang Y (2017) Multiband multi-echo imaging of simultaneous oxygenation and flow timeseries for resting state connectivity. PLoS One 12:e0169253. CrossRef Medline

Cox RW (2012) AFNI: what a long strange trip it's been. Neuroimage 62: 743-747. CrossRef Medline

Cox RW, Chen G, Glen DR, Reynolds RC, Taylor PA (2017) fMRI clustering and false-positive rates. Proc Natl Acad Sci U S A 114:E3370-E3371. CrossRef Medline

Craddock RC, James GA, Holtzheimer PE 3rd, Hu XP, Mayberg HS (2012) A whole brain fMRI atlas generated via spatially constrained spectral clustering. Hum Brain Mapp 33:1914-1928. CrossRef Medline

Craig AD (2002) How do you feel? Interoception: the sense of the physiological condition of the body. Nat Rev Neurosci 3:655-666. CrossRef Medline

Damoiseaux JS, Rombouts SA, Barkhof F, Scheltens P, Stam CJ, Smith SM,
Beckmann CF (2006) Consistent resting-state networks across healthy subjects. Proc Natl Acad Sci U S A 103:13848-13853. CrossRef Medline

Desmet C, Fias W, Hartstra E, Brass M (2011) Errors and conflict at the task level and the response level. J Neurosci 31:1366-1374. CrossRef Medline

Dosenbach NU, Nardos B, Cohen AL, Fair DA, Power JD, Church JA, Nelson SM, Wig GS, Vogel AC, Lessov-Schlaggar CN, Barnes KA, Dubis JW, Feczko E, Coalson RS, Pruett JR Jr, Barch DM, Petersen SE, Schlaggar BL (2010) Prediction of individual brain maturity using fMRI. Science 329: 1358-1361. CrossRef Medline

Duncan J (2010) The multiple-demand (MD) system of the primate brain: mental programs for intelligent behaviour. Trends Cogn Sci 14:172-179. CrossRef Medline

Duncan J, Owen AM (2000) Common regions of the human frontal lobe recruited by diverse cognitive demands. Trends Neurosci 23:475-483. CrossRef Medline

Ester M, Kriegel H-P, Sander J, Xu X (1996) A density-based algorithm for discovering clusters in large spatial databases with noise. In: Proceedings of the second international conference on knowledge discovery and data mining (Simoudis E, Han J, Fayyad U, eds), pp 226-231. Palo Alto, CA: Association for the Advancement of Artificial Intelligence.

Fair DA, Dosenbach NU, Church JA, Cohen AL, Brahmbhatt S, Miezin FM, Barch DM, Raichle ME, Petersen SE, Schlaggar BL (2007) Development of distinct control networks through segregation and integration. Proc Natl Acad Sci U S A 104:13507-13512. CrossRef Medline

Fischl B (2012) FreeSurfer. Neuroimage 62:774-781. CrossRef Medline

Friston KJ, Tononi G, Sporns O, Edelman GM (1995) Characterising the dimensionality of neuronal interactions. Hum Brain Mapp 3:302-314. CrossRef

Garrett DD, Kovacevic N, McIntosh AR, Grady CL (2011) The importance of being variable. J Neurosci 31:4496-4503. CrossRef Medline

Giedd JN, Blumenthal J, Jeffries NO, Castellanos FX, Liu H, Zijdenbos A, Paus T, Evans AC, Rapoport JL (1999) Brain development during childhood and adolescence: a longitudinal MRI study. Nat Neurosci 2:861-863. CrossRef Medline

Gilmore JH, Schmitt JE, Knickmeyer RC, Smith JK, Lin W, Styner M, Gerig G, Neale MC (2010) Genetic and environmental contributions to neonatal brain structure: a twin study. Hum Brain Mapp 31:1174-1182. CrossRef Medline

Gu S, Satterthwaite TD, Medaglia JD, Yang M, Gur RE, Gur RC, Bassett DS (2015) Emergence of system roles in normative neurodevelopment. Proc Natl Acad Sci U S A 112:13681-13686. CrossRef Medline

Habas C, Kamdar N, Nguyen D, Prater K, Beckmann CF, Menon V, Greicius MD (2009) Distinct cerebellar contributions to intrinsic connectivity networks. J Neurosci 29:8586-8594. CrossRef Medline

Koechlin E (2011) Frontal pole function: what is specifically human? Trends Cogn Sci 15:241. CrossRef Medline

Koechlin E, Ody C, Kouneiher F (2003) The architecture of cognitive control in the human prefrontal cortex. Science 302:1181-1185. CrossRef Medline

Krienen FM, Tu PC, Buckner RL (2010) Clan mentality: evidence that the medial prefrontal cortex responds to close others. J Neurosci 30:1390613915. CrossRef Medline

Kundu P, Inati SJ, Evans JW, Luh WM, Bandettini PA (2012) Differentiating BOLD and non-BOLD signals in fMRI time series using multi-echo EPI. Neuroimage 60:1759-1770. CrossRef Medline

Kundu P, Brenowitz ND, Voon V, Worbe Y, Vértes PE, Inati SJ, Saad ZS, Bandettini PA, Bullmore ET (2013) Integrated strategy for improving functional connectivity mapping using multiecho fMRI. Proc Natl Acad Sci U S A 110:16187-16192. CrossRef Medline

Kundu P, Benson BE, Baldwin KL, Rosen D, Luh WM, Bandettini PA, Pine DS, Ernst M (2015) Robust resting state fMRI processing for studies on typical brain development based on multi-echo EPI acquisition. Brain Imaging Behav 9:56-73. CrossRef Medline

Kundu P, Voon V, Balchandani P, Lombardo MV, Poser BA, Bandettini PA (2017) Multi-echo fMRI: a review of applications in fMRI denoising and analysis of BOLD signals. Neuroimage 154:59-80. CrossRef Medline

Lebel C, Walker L, Leemans A, Phillips L, Beaulieu C (2008) Microstructural maturation of the human brain from childhood to adulthood. Neuroimage 40:1044-1055. CrossRef Medline

Lieberman MD (2007) Social cognitive neuroscience: a review of core processes. Annu Rev Psychol 58:259-289. CrossRef Medline 
Murphy D, Spooren W (2012) EU-AIMS: a boost to autism research. Nat Rev Drug Discov 11:815-816. CrossRef Medline

Owen AM, Stern CE, Look RB, Tracey I, Rosen BR, Petrides M (1998) Functional organization of spatial and nonspatial working memory processing within the human lateral frontal cortex. Proc Natl Acad Sci U S A 95: 7721-7726. CrossRef Medline

Parsons LM, Denton D, Egan G, McKinley M, Shade R, Lancaster J, Fox PT (2000) Neuroimaging evidence implicating cerebellum in support of sensory/cognitive processes associated with thirst. Proc Natl Acad Sci U S A 97:2332-2336. CrossRef Medline

Passingham RE, Wise SP (2012) The neurobiology of the prefrontal cortex: anatomy, evolution, and the origin of insight: Oxford Psychology Series, No. 50. New York, NY: Oxford UP.

Paus T, Collins DL, Evans AC, Leonard G, Pike B, Zijdenbos A (2001) Maturation of white matter in the human brain: a review of magnetic resonance studies. Brain Res Bull 54:255-266. CrossRef Medline

Pedregosa F, Varoquaux G, Gramfort A, Michel V, Thirion B, Grisel O, Blondel M, Prettenhofer P, Weiss R, Dubourg V (2011) Scikit-learn: machine learning in python. J Mach Learn Res 12:2825-2830.

Petrides M (1995) Impairments on nonspatial self-ordered and externally ordered working memory tasks after lesions of the mid-dorsal part of the lateral frontal cortex in the monkey. J Neurosci 15:359-375. Medline

Petrides M (2000) The role of the mid-dorsolateral prefrontal cortex in working memory. Exp Brain Res 133:44-54. CrossRef Medline

Petrides M (2005) Lateral prefrontal cortex: architectonic and functional organization. Philosophical Transactions of the Royal Society B: Biological Sciences 360:781-795. CrossRef Medline

Poser BA, Versluis MJ, Hoogduin JM, Norris DG (2006) BOLD contrast sensitivity enhancement and artifact reduction with multiecho EPI: parallel-acquired inhomogeneity-desensitized fMRI. Magn Reson Med 55:1227-1235. CrossRef Medline

Posse S, Wiese S, Gembris D, Mathiak K, Kessler C, Grosse-Ruyken ML, Elghahwagi B, Richards T, Dager SR, Kiselev VG (1999) Enhancement of BOLD-contrast sensitivity by single-shot multi-echo functional MR imaging. Magn Reson Med 42:87-97. CrossRef Medline

Power JD, Barnes KA, Snyder AZ, Schlaggar BL, Petersen SE (2012) Spurious but systematic correlations in functional connectivity MRI networks arise from subject motion. Neuroimage 59:2142-2154. CrossRef Medline

Power JD, Cohen AL, Nelson SM, Wig GS, Barnes KA, Church JA, Vogel AC, Laumann TO, Miezin FM, Schlaggar BL, Petersen SE (2011) Functional network organization of the human brain. Neuron 72:665-678. CrossRef Medline

Raz N (2004) The aging brain: structural changes and their implications for cognitive aging. In: New frontiers in cognitive aging (Dixon R, Backman L, Nilsson L-G, eds), pp 115-134. New York, NY: Oxford UP.

Raz N, Torres IJ, Spencer WD, White K, Acker JD (1992) Age-related regional differences in cerebellar vermis observed in vivo. Arch Neurol 49:412-416. CrossRef Medline

Reis DJ, Golanov EV (1997) Autonomic and vasomotor regulation. Int Rev Neurobiol 41:121-149. CrossRef Medline

Rubinov M, Sporns O (2010) Complex network measures of brain connectivity: uses and interpretations. Neuroimage 52:1059-1069. CrossRef Medline

Schmahmann JD, Caplan D (2006) Cognition, emotion and the cerebellum. Brain 129:290-292. CrossRef Medline

Schmahmann JD, Sherman JC (1998) The cerebellar cognitive affective syndrome. Brain 121:561-579. CrossRef Medline

Scholkopf B, Sung KK, Burges CJC, Girosi F, Niyogi P, Poggio T, Vapnik V (1997) Comparing support vector machines with gaussian kernels to radial basis function classifiers. IEEE Trans Signal Process 45:2758-2765. CrossRef

Singer T, Seymour B, O’Doherty J, Kaube H, Dolan RJ, Frith CD (2004) Empathy for pain involves the affective but not sensory components of pain. Science 303:1157-1162. CrossRef Medline

Stoodley CJ, Schmahmann JD (2009) Functional topography in the human cerebellum: a meta-analysis of neuroimaging studies. Neuroimage 44: 489-501. CrossRef Medline

Stoodley CJ, Valera EM, Schmahmann JD (2012) Functional topography of the cerebellum for motor and cognitive tasks: an fMRI study. Neuroimage 59:1560-1570. CrossRef Medline

Supekar K, Musen M, Menon V (2009) Development of large-scale functional brain networks in children. PLoS Biol 7:e1000157. CrossRef Medline

van den Heuvel MP, Hulshoff Pol HE (2010) Exploring the brain network: a review on resting-state fMRI functional connectivity. Eur Neuropsychopharmacol 20:519-534. CrossRef Medline

von Economo CF, Koskinas GN (1926) Die cytoarchitektonik der hirnrinde des erwachsenen menschen. J. Springer 16: 816. CrossRef

Wallace GL, Eric Schmitt J, Lenroot R, Viding E, Ordaz S, Rosenthal MA, Molloy EA, Clasen LS, Kendler KS, Neale MC, Giedd JN (2006) A pediatric twin study of brain morphometry. J Child Psychol Psychiatry 47: 987-993. CrossRef Medline

Zhang H, Schneider T, Wheeler-Kingshott CA, Alexander DC (2012) NODDI: practical in vivo neurite orientation dispersion and density imaging of the human brain. Neuroimage 61:1000-1016. CrossRef Medline 